\title{
The Fractal Nature of Implicit Knowledge*
}

\author{
Mikhail Oseledchik \\ Department of philosophy and social sciences \\ High school of printing and media industry \\ Moscow Polytechnic University \\ 38 Bolshaya Semenovskaya Street, Moscow, 107023, Russia \\ E-mail: balu13@yandex.ru
}

\author{
Marina Ivleva \\ Faculty of Humanities and Social Sciences \\ Department of Social Philosophy \\ Peoples' Friendship University of Russia (RUDN \\ University) \\ 6 Miklukho-Maklaya Street, Moscow, 117198, Russia \\ E-mail: marinanonna@yandex.ru
}

\author{
Vitaly Ivlev \\ National Research University \\ Moscow State Technical University named after N.E. \\ Bauman (MSTU named after N.E.Bauman) \\ 5/1 2d Baumanskaya Street, Moscow, 105005, Russia \\ E-mail: vitalijivlev@yandex.ru
}

\begin{abstract}
The article analyzes the fractal nature of implicit knowledge and its significance for understanding the nature of transmission and reconfiguration of knowledge. Implicit knowledge is one of the ways of existence of consciousness. It lies at the basis of a person's professional abilities. Forming a message by its creator is the process of building a self-similar structure - a fractal, i.e. an articulated construction, similar to those conceptual schemes that are contained in the consciousness of the subject. It means that the message is the transfer of this self-similar structure - the fractal - to the addressee. By receiving the message and decoding it, the addressee builds in his mind the mental construction, which in its turn is similar to the semantic construction which is embedded in the message, i.e. understanding the fractal.
\end{abstract}

Keywords-Implicit knowledge; stochastic fractal; reconfiguration of knowledge; knowledge transfer; knowledge management systems

\section{INTRODUCTION}

The beginning of attention to the study of the nature of implicit knowledge was laid after the appearance of M.Polanyi's classical work "Personal knowledge". Implicit knowledge is one of the ways of existence of consciousness, it is an unformed way of its existence, a kind of peripheral knowledge, due to which this knowledge is difficult to transfer to another subject. Often this knowledge can be transferred only through personal communication and training, through the development of personal experience of some specific activity. This knowledge can be acquired unintentionally and unconsciously, but the comprehension and solution of the problems facing the specialist depend on these schematically organized knowledge [1] and skills

*This paper was financially supported by the Ministry of Education and Science of the Russian Federation on the program to improve the competitiveness of Peoples' Friendship University (RUDN University) among the world's leading research and education centers in the 2016-2020 (The Agreement number 02.A03.21.0008). managed at the unconscious level.

\section{THE CONCEPT OF 'IMPLICIT KNOWLEDGE'}

Implicit knowledge can be objectified in non-linguistic ways - first of all, in the ways of activity, in gestures, facial expressions, dances and painting.

Implicit knowledge is the prerequisite and reason for a person's daily activities, it forms the implied sense of spoken words, it is the prerequisites and conditions for communication, cognition and understanding between people. "It is based on traditions, customs, everyday habits and simple common sense. It consists of beliefs, ideals, values, schemes and mental models that are deeply rooted in us, and which we often take for granted. However hard it is formed, this implicit knowledge forms our perception of the world" [2].

Implicit knowledge is non-articulated and unreflected personal knowledge and personal experience, so any codification always happens with a partial loss of its content. This is primarily the skills, competence, abilities and feelings of a person.

Implicit knowledge is woven into human activity, it is the basis of professional automatisms, secrets of mastery, insights and intuitions.

Implicit knowledge is an important feature of social networks, it is contained in them and transmitted through informal lateral communication between members of the community.

There is a peculiar layer of implicit ontological prerequisites (three-dimensional space, the unity of the world, the laws of logic), which gives the subject an understanding of the world as a whole. This is the basis for forming the knowledge of a particular person. The similarity of the 
anatomical and physiological characteristics of the subjects of cognition gives the similarity to the course of psychological reactions.

In the article "Understanding Practical Intelligence" three key features of the notion of implicit knowledge are highlighted: "It characterizes the circumstances under which knowledge is obtained, its cognitive structure and application. First, implicit knowledge, as a rule, arises independently, without any reinforcement by external circumstances or with very little reinforcement (i.e. rather due to one's own experience, and not as a result of special training). Secondly, implicit knowledge is considered procedural in its nature. It is associated with a specific application in specific situations or in a class of situations. Thirdly, due to the fact that most of the knowledge is obtained on the basis of their own experience, it is of practical importance only for the person who possesses it" [3].

There are classifications of implicit knowledge, considered in detail by E.O. Gubanova in the article "Implicit knowledge: essence and types" [4].

Proceeding from the belonging of implicit knowledge to a certain type of subject, G.Starikova subdivides implicit knowledge into:

- personal - a lot of unconceptualized, irrationallypersonal, implicit, profound phenomena, not verbalized at this stage of the development of science;

- paradigmatic - implicit knowledge belonging to a certain scientific community (these are logical and linguistic rules and norms, the generally accepted language of science, well-known fundamental laws and principles, philosophical and worldview assumptions, the scientific picture of the world, the style of thinking);

- objective (intersubjective) knowledge - knowledge that is fixed in a certain historical stage in a symbolic or another form (for example, common symbols in a given cultural context);

- transsubjective - hidden, extremely general grounds of cognitive activity, universal ideals and norms, without which the development of the phenomenon of cognition is impossible [5].

Of particular interest to us is the so-called background knowledge: "Background knowledge is a mutual knowledge of the realities by a speaker and a listener, which is the basis of language communication. Background knowledge forms the part of what sociologists call mass culture, it represents information that is certainly known to all members of the community" [6].

Implicit knowledge, therefore, is very heterogeneous and, is, naturally, unevenly distributed among carriers, which causes the need for its rearrangement and distribution. But this is precisely one of the fundamental tasks of modern knowledge management systems.

\section{FRACTAL CHARACTERISTIC OF IMPLICIT KNOWLEDGE}

The communication act is always divided into several stages:

- the emergence of the situation of the need for a communication act (meaning that a question can be, or should be asked, to which an answer should be given, or a request for help should be voiced, or the need to help or show something or teach something is understood);

- awareness by the sender that the situation of the need for a communication act has really arisen;

- awareness by the sender of the meaning and content of this need (roughly speaking, awareness of the meaning of the request);

- $\quad$ forming the request by the sender;

- $\quad$ encoding the message based on the request;

- $\quad$ articulation - sending the message to the addressee;

- $\quad$ receiving the message by the addressee;

- $\quad$ its decoding;

- its understanding;

- $\quad$ response of the addressee;

- $\quad$ forming a response;

- encoding the message;

- transfer it to the sender;

- decoding the message;

- clarifying its meaning.

In a real communication process, all these stages pass almost instantly, but in fact they are always present and are based on common explicit and implicit linguistic knowledge. The possibility of a communication act is in principle based on the similarity of the linguistic competences of the creator and the addressee of the message [7]. Forming a message by its creator is the process of building a self-similar structure a fractal, i.e. an articulated construction, similar to those conceptual schemes that are contained in the consciousness of the subject. It means that a message is the sounding of the subject's knowledge and the transfer of this self-similar structure - the fractal - to the addressee.

Receiving the message and decoding it, the addressee builds in his mind, again, the mental construction, which in its turn is similar to the semantic construction that is embedded in the message, i.e. it is the understanding of the fractal.

The classical models of transferring knowledge at the level of instinct are based on the interpretation of translation and the assimilation of knowledge as a simple linear process. In fact, the transfer of knowledge is not a simple linear process due to the fact that the recipient of knowledge already has his own system of explicit and implicit 
knowledge, has his stock of meanings, his worldly experience, the level of language proficiency, the level of intelligence development and other important personal characteristics. This system at every stage of a person's life is balanced and develops dynamically. The fact of getting new information causes a violation of this equilibrium, new information may be a foreign body for this established system of the inner world of a person, which, frankly speaking, most often happens. The new received message is acquired by the recipient with the help of a kind of a filter of the cognitive system already existing in him [8].

The system of human knowledge as an indispensable product of the work of his memory is the result of the process of self-organization and it lives by nonlinear laws, therefore its behavior is often unpredictable, which becomes the source of the unexpected reconfiguration of knowledge, which, in its turn, is ambiguous. It is not constructive, due to the fact that it is almost impossible to transfer knowledge accurately and in full. And at the same time, it is constructive, as it gives creative reconfiguration and increment of knowledge.

So, in order to achieve the assimilation of knowledge, that is, the reproduction of knowledge of the information carrier in the system of knowledge of the information receiver in the most complete and accurate, i.e. similar, manner, we can use the interpretation of knowledge as a fractal - a self-similar structure whose image does not depend on the scale, being a recursive dynamic model, each part of which repeats in its development the development of the whole model.

Considering that both the carrier and the recipient of knowledge have their own system of knowledge, and that the knowledge of each individual is a self-organizing self-similar structure, because both the carrier and the recipient have a lot of common knowledge: a single system of values, a common language, a single lexico-semantic structure of this language, the standard logic of reasoning, the ways and styles of thinking, the general stock of everyday skills, a sense of humor, even developed and learned with varying degrees, but still similar to each other due to the existence of new knowledge of a single socio-cultural paradigm in the carrier and addressee - we can state that knowledge has a fractal nature.

The hidden knowledge of each individual can be interpreted as a stochastic fractal. Each person has a basic fractal, which forms the core of his worldview and relationship with the world. As a person develops, this basic fractal becomes more complicated, a person develops with time the patterns of repetitive reactions, i.e. he develops a certain algorithm for arranging events in a certain drawing. And this is facilitated by the awareness of life within the framework of a fractally organized natural environment.

Implicit knowledge is a system of fractals overlapping each other at the same time.

Implicit background knowledge is an intersubjective base fractal, heavily dependent on the national, cultural and social characteristics of the human environment. Primary socialization of a person is the process of assimilating by a child this basic fractal, i.e. the structure, which includes elements of linguistic competence, the rules of thinking, the traditions of behavior, the basic ideas about the structure of the world, ethical and aesthetic attitudes. The basic fractal gives a person standard methods and criteria for evaluation, which in the early stages of human development are not analyzed and are not questioned. Accordingly, the behavioral responses of the person are again built into certain inherited patterns, the alignment of peripheral fractals is underway.

Further individual development of a person as a personality is a process of complicating the initial base fractal, which absorbs additional elements of implicit knowledge due to the intellectual and physiological characteristics of the individual, the speed and effectiveness of his thinking, his creative abilities, the level of learning and the specifics of his personal experience, including emotional experience, and the sphere of his activity. This complication of the base fractal leads to heterogeneity of implicit knowledge and skills, to their uneven distribution among different people. This is the main reason for the need for training, i.e. for the transfer of individual experience, skills and abilities from one carrier of implicit knowledge to another.

Implicit knowledge of the person forms a new individual fractal, determining the everyday, professional and intellectual behavior of this subject. In a complex interference pattern of fractals of implicit knowledge there actively participate, in addition to the basic and individual fractals, the fractals of paradigmal implicit knowledge, fractals, which form a way and style of thinking and practical activity of an individual, who exists within the framework of a certain micro-group (including organization).

When receiving a message, the addressee decodes its direct meaning quite quickly, since he does this on the basis of an intersubjective base fractal. But the assimilation of the message and the identification of its additional meanings directly depends on the individual fractal of the personality and on the fractal of the implicit knowledge which belongs to the microgroup within which a particular person lives and professionally realizes. Reconfiguration of the received knowledge is absolutely individual and is possible only if the addressee has specific individual creative abilities and special elements of explicit and implicit knowledge.

Knowledge of each specific individual is conceptual, it is a fractal in relation to the concept sphere of the society as a whole.

The assimilation of knowledge by the individual and its reconfiguration is influenced by a number of attractors, the number, power and directivity of which are practically impossible to trace, hence the possible reconfiguration and production of new knowledge is almost impossible to calculate.

Knowledge of the individual can be systematized only partially, depending on the possibility of its fundamental codification [6], the rest of the array of knowledge is contained in the form of unencoded elements, in particular, 
in the form of practical skills and abilities, intuitions, metaphors, certain emotional images, but at the same time all these elements of knowledge are closely related to each other by multiple semantic series, associative, logical and other chains, which, if necessary, "enable" instantaneous actualization of necessary knowledge.

Knowledge is dialogical: it is always a product of the internal and external dialogue of the carrier of knowledge with its addressee in the course of the transfer of knowledge and in the process of its individual rethinking [9].

Knowledge is communicative [10]: in the process of communication, it is more accurately assimilated by the individual; for the more efficient transfer of his knowledge, the subject also reconfigures it, turning it into a more systematized, precise and understandable for the addressee.

The assimilation and understanding of the knowledge which is being acquired by the subject depends on the level of discipline and training of his thinking, on the general level of culture and education, on the standards of the accepted logic, on the social orientation of the subject, on the impact on the process of assimilation of sets of attractors. The depth and correctness of understanding are not the result of a linear process of consuming knowledge [11]. We can never with a high degree of accuracy perceive the knowledge of another individual due to a great number of small circumstances that influence the way of understanding and revealing certain semantic units. The interpretation of knowledge as a stochastic fractal allows us to be loyal to the fact that the knowledge transmitted by the carrier, which consists of the elements $\{\mathrm{ABCDEF}\}$, will be reproduced as a self-similar system $\{\mathrm{A} 1 \mathrm{~B} 1 \mathrm{C} 1 \mathrm{D} 1 \mathrm{E} 1 \mathrm{~F} 1\}$.

The ability to reconfigure the acquired knowledge and its increment by the subject depends on the degree of his creativity and the measure of freedom from the usual cultural schematisms in favor of new inversion options, so it is almost impossible to calculate and predict new results and discoveries that a particular individual will make. But with a high degree of probability, one can predict the possibility that a particular subject is capable of obtaining new results and discoveries, and can also make a good career.

\section{CONCLUSION}

The transfer of knowledge is at first a process of fractal destruction, as being received new knowledge destroys the existing cognitive system of the recipient and is perceived and interpreted by him incompletely and inaccurately. Then, because of the self-similarity of the cognitive systems of different individuals living and thinking in a single lexicosemantic space, the process of assembling a fractal begins: the obtained constituent parts of new knowledge are combined into single formulas and are perceived more or less adequately and isomorphically to the original message. New knowledge of the recipient turns into a fractal knowledge of the addressee. But then comes the most interesting and important stage: the process of reconfiguring knowledge, when thanks to the presence of a whole set of hard-to-recognize personal attractors of the recipient, knowledge begins to be reinterpreted and reconsidered. The result of this reconfiguration is a creatively enriched knowledge.

The assimilation of these new meanings of knowledge entails the reorganization of the system of knowledge of the carrier and its transformation into a fractal of the knowledge of the recipient. And this, in its turn, leads to the transformation of the entire cognitive paradigm, uniting both the carrier and the recipient of knowledge.

\section{REFERENCES}

[1] Oseledchik M., Inozemtsev V., Ivlev V., Ivleva M. LogicalPhilosophical Approach to the Interpretation of the Concept of Knowledge //Proceedings of the 2016 3rd International Conference on Education, Language, Art and Inter-cultural Communication (ICELAIC 2016). Advances in Social Science, Education and Humanities Research. Paris: Atlantis Press, 2017. Volume 40. P.297301

[2] [Electronic resource]. The access point http://infoarm.ru/alphabet_index/n/neyavnoe-znanie.html.-11.02.2017.

[3] Understanding practical intelligence [Electronic resource]. The access point https://murzim.ru/psihologija/prakticheskij-intellekt/10933ponimanie-prakticheskogo-intellekta.html - 05.12.2016.

[4] Gubanova E.O. Implicit knowledge: essence and types http://cyberleninka.ru/article/n/neyavnoe-znanie-suschnost-i-vidy 07.12.2016.

[5] Starikova G.G. The nature and epistemological functions of personal implicit knowledge: dis.... cand.philosoph. sciences. Kharkov, 2001.

[6] Inozemtsev V.A., Ivlev V.Yu, Ivleva M.L., Oseledchik M.B. Representology in the System of Modern Epistemology //Proceedings of the 2016 3rd International Conference on Education, Language, Art and Inter-Cultural Communication (ICELAIC 2016). Advances in Social Science, Education and Humanities Research. Paris: Atlantis Press, 2017. Volume 40. P. 697-701

[7] Chistyakov D.I. Social Dimension of Media Space in the Age of Postmodernity. In the Context of Objective Knowledge Obtainment // Proceedings of the 2016 International Conference on Arts, Design, and Contemporary Education. Volume 64. (ICADCE 2016). Advances in Social Science, Education and Humanities Research". Atlantis Press. - Paris. France. 2016. ISSN 2352-5398, volume 64. P. 297-300

[8] Chistyakova O.V. The "Other" Culture Formation in Russia. The View Through the Informational Society Theory and Actual Process of External Migration // Proceedings of the 2016 International Conference on Arts, Design, and Contemporary Education. Volume 64. (ICADCE 2016). Advances in Social Science, Education and Humanities Research". Atlantis Press. - Paris. France. 2017. ISSN 2352-5398, volume 64. P. 626-631.

[9] Ivlev V.Yu., Ivleva M.L., Inozemtsev V.A., Udovik V.E. Informational society and formation of a new epistemological paradigm in modern science. Moscow, 2013

[10] Ivlev V.Yu., Barkova E.V., Ivleva M.I., Buzskaya O.M. Environmental approach to the study of the modern stage of information society development: research prospects // International Journal of Environmental and Science Education. 2016, v. 11, № 16 , pp. 9113-9124.

[11] Chistyakova O.V. Philosophical-Anthropological Meanings of Postmodernism: "Mediatizing" Human // Proceedings of the International Conference on Contemporary Education, Social Sciences and Humanities. Advances in Social Science, Education and Humanities Research (ICCESSH 2016). Volume 74. ISBN (on-line): 978-94-6252-215-2. Paris: Atlantis Press, 2016. P. 637-642. 